\title{
Long-term potentiation in hippocampus involves sequential activation of soluble guanylate cyclase, cGMP-dependent protein kinase and CGMP-degrading phosphodiesterase, alterations in hyperammonemia Pilar Monfort* and Vicente Felipo
}

Address: Laboratory of Neurobiology, Fundación Valenciana de Investigaciones Biomédicas, Valencia, Spain

Email: Pilar Monfort* - pili@ochoa.fib.es

* Corresponding author

from 2nd International Conference of cGMP Generators, Effectors and Therapeutic Implications

Potsdam, Germany, I0-12 June, 2005

Published: 16 June 2005

BMC Pharmacology 2005, 5(Suppl I):P66 doi:I0.II86/I47I-22I0-5-SI-P66

Long-term potentiation (LTP) is a long-lasting enhancement of synaptic transmission efficacy and is considered the base for some forms of learning and memory.

Hyperammonemia is considered the main responsible for the neurological alterations found in liver disease and hepatic encephalopathy, including decreased intelectual and cognitive function. Ammonia affects both excitatory and inhibitory synaptic transmission in the mammalian brain by a variety of mechanisms. LTP is impaired in hyperammonemia and this may contribute to the impairment of cognitive function. However the mechanism by which hyperammonemia impairs LTP remains unclear. We have demnostrated that a sequential activation of soluble guanylate cyclase (sGC), cGMP-dependent protein kinase (PKG) and cGMP-degrading phosphodiesterase (PDE) is necessary for proper induction of LTP.

Application of the tetanus induces a rapid rise in cGMP, reaching a maximum 10 seconds after the tetanus. cGMP decreased below basal levels $5 \mathrm{~min}$ after the tetanus remaining low at least until $60 \mathrm{~min}$. The activity of sGC increase at 10 seconds after tetanus remaining high at 5 min and returning to basal levels at $60 \mathrm{~min}$. after the tetanus. This explains the initial transient increase in cGMP. The subsequent decrease in cGMP is due to sustained tetanus-induced increase in the activity of cGMP-degrading phosphodiesterase, which remains activated $60 \mathrm{~min}$. after tetanus. We studied the mechanism by which the tetanus induces the activation of PDE. Tetanus-induced activation of PDE and decrease of cGMP were prevented by inhibit- ing PKG. This indicates that the initial increase in cGMP activates PKG that phosphorylates (and activates) PDE, which, in turn, degrades CGMP. Inhibition of any step of this pathway impairs LTP, indicating that proper induction of LTP involves activation of this pathway.

We found that hyperammonemia impairs LTP in hippocampus by altering the modulation of this sGC-PKG-cGMP degrading PDE pathway. Exposure of hippocampal slices to $1 \mathrm{mM}$ ammonia completely prevents tetanus-induced decrease of cGMP by imparing PKG-mediated activation of cGMP-degrading phosphodiesterase. LTP is also impaired in hippocampal slices from hyperammonemic rats without liver failure and from rats with portacaval anastomosis, an experimental model of hepatic failure. The impairment is more severe in rats with portacaval anastomosis. These alterations in LTP may be involved in the cognitive impairment in patients with hepatic encephalopathy. 